Original Article

\title{
DRUG UTILIZATION PATTERN IN PAEDIATRIC PATIENTS IN A SECONDARY CARE HOSPITAL
}

\section{R. SENTHILSELVI ${ }^{*}$, M. BOOPANA', LINTA SATHYAN1, PRIYA VISUVASAM², V. GANESAN1}

${ }^{1}$ Department of Pharmacy Practice, The Erode College of Pharmacy, Erode, 638112, Tamilnadu, India, ${ }^{2}$ Govt. Head Quarters Hospital, Tiruppur, Tamilnadu, India

Email: Senthilselvi65@gmail.com

Received: 12 Nov 2018 Revised and Accepted: 22 Feb 2019

\section{ABSTRACT}

Objective: The study was carried out to generate research on drug utilization pattern in paediatric patients in government headquarters hospital, Tiruppur, Tamilnadu, India using world health organization (WHO) and international network for rational use of drugs (INRUD) core indicator.

Methods: The core prescribing indicators of the world health organization was used to assess the appropriate use of drugs. Index of rational drug prescribing (IRDP) developed by Zhang and Zhi was also used to find out the performance of a health care system in terms of drug utilization.

Results: Out of 859 drugs in 200 cases the highest prescribed drug class is antibiotics 304(35.39\%) and the majority of drugs were administered in injectable form 412(47.94\%). It was analysed that a majority of prescriptions $117(58.5 \%)$ were discharged in between $4-7$ d. $161(80.5 \%)$ patients were dismissed after completion of treatment. On analysing world health organization (WHO) prescribing core indicators, the average number of drugs per prescription was 4.29 which is higher than double the average number (i.e., 2). This indicates poly-pharmacy practice. $97.78 \%$ of drugs were prescribed were the generic name and percentage of encounters with antibiotic prescribed was $90.5 \%$ which is thrice greater than world health organization (WHO) standard $<30 \%$. Prescribing of injections is not within the world health organization (WHO) recommended range<20\% and it was far higher showing $97.5 \%$ which is essential for paediatric inpatients. The prescribing practice in paediatric ward is in complete adherence to national essential drug list (EDL) or formulary. The mean value of the index of rational drug prescribing (IRDP) obtained was 3.09 which is very similar to that of optimal value world health organization (WHO).

Conclusion: Prescription by generic name, prescribing drugs from essential drug list (EDL) and free government supply are encouraging findings in this hospital. The result shows poly-pharmacy and overuse of antibiotics are the areas to be concerned. The better clinical outcome shows rational prescribing is practised well.

Keywords: Core indicators, an International network for rational use of drugs (INRUD), Index of Rational Drug Prescribing (IRDP), Poly-pharmacy, Essential drug list (EDL), Antibiotics

(C) 2019 The Authors. Published by Innovare Academic Sciences Pvt Ltd. This is an open access article under the CC BY license (http://creativecommons.org/licenses/by/4.0/] DOI: http://dx.doi.org/10.22159/ijpps.2019v11i4.30746

\section{INTRODUCTION}

World Health Organization (WHO) defined drug utilization research as 'studies on the marketing, distribution, prescription and the use of drugs in a society, with special emphasis on the resulting medical, social and economic consequences [1, 2]. Drug utilization studies are important in order to evaluate the appropriate and judicious use of drug therapy [3]. Appropriate drug utilization studies are properly utilized to evaluate the efficacy, safety, convenience and economic aspects at all levels in the chain of drug use.

Various drug utilization reviews have been conducted all over the world but only limited studies are conducted addressing the drug use pattern in paediatric population especially in India [4]. 20 to $25 \%$ of total world population comprise paediatric, which are more prone to acute and chronic infectious disease. The incomplete development of paediatric physiological conditions and their rapid growth and development need some accurate diagnosis and evidence-based medical treatment [5].

Infants and children are the most vulnerable population groups. They are particularly sensitive to contract illness and harmful effects of drugs because of their difference in pharmacokinetics and pharmacodynamics to that of adults [6]. Also, drug use in paediatric is not extensively researched and the dosage form of licensed drugs is limited [7].

Monitoring the safety of medical use in children is of paramount importance because only a limited data can be generated through clinical trials of drug development [8]. Infants and children constitute a large population in a developing country like India. Promotion of appropriate and safe drugs for children is hour globally essential [9].
Doses for children often derived from adult dosages after adjusting the body weight. But even within the paediatric population especially preterm neonate doses varies from the calculated weight-adjusted doses [10]. There are likewise various drug-related problems in children such as heterogeneous nature of paediatric population, offlabel and unlicensed drug use, lack of familiarity between adult and paediatric guidelines, inability to swallow solid dosage form etc [11]. Prescription auditing, a type of vigilance activity, which is beneficial in clinical practice for reducing the burden of disease and to ensure irrational prescribing is hence indispensable [9].

\section{MATERIALS AND METHODS}

\section{Study site}

The study was conducted in the department of child health on Government district headquarters Hospital, Tiruppur, a 500 bedded hospital.

\section{Study design}

This is a prospective observational study and was carried out in the department of child health unit of Government Headquarters Hospital, Tiruppur.

\section{Study period}

The study was undertaken during a period of 6 mo from March 2018 to August 2018.

\section{Study population}

The study population includes the patients of Govt. Headquarters Hospital, Tiruppur. A total of 200 numbers of admitted cases were chosen from paediatric ward. 


\section{Ethical approval}

Research protocol, data collection form and informed consent form (Tamil/English) was approved by the Institutional Human Ethics Committee (IHEC) of the Government Headquarters Hospital, Tiruppur, Tamilnadu (IHEC NO: IHEC/GHTiruppur/ECP/PD-002). The approval was obtained before the study was commenced.

\section{Patient selection}

Patient's selection was based on the inclusion criteria. The inclusion criteria included newborn of $1 \mathrm{mo}$ to $12 \mathrm{y}$, considered both male and female patients who stayed in the hospital for more than $24 \mathrm{~h}$. Exclusion criteria included all adult patients, outpatients, intensive care unit emergency patients, immunocompromised patients and the patients not willing to participate in the study.

\section{Prescribing core indicators}

The prescribing core indicators of WHO was an average number of medicines per encounter, percentage of medicines prescribed by generic name, percentage of encounters with an antibiotic prescribed, percentage of encounters with an injection prescribed and percentage of medicines prescribed from the essential drug list formulary.

Index of rational drug prescribing (IRDP) was also performed.

\section{Statistical analysis}

Data were entered and analysed using Microsoft Excel (windows 8; version 2007). Graphical representation is used for visual interpretation of the analysed data.

\section{RESULTS}

Out of 200 prescriptions analyzed, a total of 859 drugs were prescribed.

Table 1: Age-wise classification

\begin{tabular}{lll}
\hline Age group & Frequency & Percentage (\%) \\
\hline $1-23$ Mo & 58 & 29 \\
$2-11$ Y & 134 & 67 \\
$12 Y$ & 8 & 4 \\
\hline
\end{tabular}

Table 2: Gender wise distribution

\begin{tabular}{lll}
\hline Age & \% of male & \% of female \\
\hline $1-23$ Mo & 29 & 29 \\
$2-11$ Y & 65 & 69 \\
$12 \mathrm{Y}$ & 6 & 2 \\
\hline
\end{tabular}

Table 3: Distribution of diseases in paediatric patients

\begin{tabular}{lll}
\hline Distribution of diseases & No. of diseases & Percentage (\%) \\
\hline Seizure disorder & 27 & 13.5 \\
GI disorder & 35 & 17.5 \\
Fever & 54 & 27 \\
Respiratory disease & 46 & 23 \\
UTI & 4 & 2 \\
Anaemia/Malnutrition & 4 & 2 \\
Poison cases & 14 & 7 \\
Genetic disorders & 2 & 1 \\
Autoimmune disease & 1 & 0.5 \\
Others & 13 & 6.5 \\
\hline
\end{tabular}

Table 4: Medicines prescribed to paediatric patients

\begin{tabular}{|c|c|c|}
\hline Medicines category & No. of drugs & Percentage (\%) \\
\hline Anti-asthmatics & 57 & 6.64 \\
\hline Anti allergics & 43 & 5.0 \\
\hline NSAIDS & 159 & 18.5 \\
\hline Anti-emetics & 41 & 4.77 \\
\hline Corticosteroids & 5 & 0.58 \\
\hline Anti-epileptics & 42 & 4.88 \\
\hline Antiulcer agents & 82 & 9.54 \\
\hline Antispasmodics & 4 & 0.47 \\
\hline Vaccines & 3 & 0.34 \\
\hline Anti-amoebic agents & 14 & 1.63 \\
\hline Vitamin supplements & 10 & 1.16 \\
\hline Antibiotics & 304 & 35.39 \\
\hline Others & 95 & 11.1 \\
\hline
\end{tabular}

Table 5: Antibiotics prescribed to paediatrics

\begin{tabular}{lll}
\hline Antibiotic classification & No. of antibiotics & Percentage (\%) \\
\hline Aminoglycosides & 83 & 27 \\
Cephalosporins & 167 & 55 \\
Penicillin & 22 & 7 \\
Penicillin combinations & 11 & 3 \\
Macrolides & 15 & 5 \\
Tetracyclines & 2 & 1 \\
Sulphonamide combinations & 2 & 1 \\
Carbapenem & 2 & 1 \\
\hline
\end{tabular}


Table 6: Route of administration

\begin{tabular}{llll}
\hline ROA & Sub-class & Frequency & Percentage (\%) \\
\hline Injectables & Intravenous & 409 & 47.6 \\
& Intramuscular & 2 & 0.23 \\
Oral & Subcutaneous & 1 & 0.11 \\
Inhale & - & 384 & 44.7 \\
Topical & - & 62 & 7.21 \\
\hline
\end{tabular}

Table 7: Drug-drug interaction per encounter

\begin{tabular}{lll}
\hline Drug interaction & No. of prescriptions & Percentage (\%) \\
\hline Cases with DI & 126 & 63 \\
Cases without DI & 74 & 37 \\
\end{tabular}

Table 8: Duration of hospital stay

\begin{tabular}{|c|c|c|}
\hline Duration & No. of prescriptions & Percentage (\%) \\
\hline $1-3 \mathrm{~d}$ & 58 & 29 \\
\hline $4-7 d$ & 117 & 58.5 \\
\hline $8-11 d$ & 22 & 11 \\
\hline 12 or above & 3 & 1.5 \\
\hline
\end{tabular}

Table 9: Patient outcomes

\begin{tabular}{lll}
\hline Patient outcomes & No. of patients \\
\hline Discharged & 161 & Percentage (\%) \\
Referred & 8 & 80.5 \\
Discharged at request & 21 & 4 \\
Absconded/leave against medical advice (LAMA) & 10 & 10.5 \\
\hline
\end{tabular}

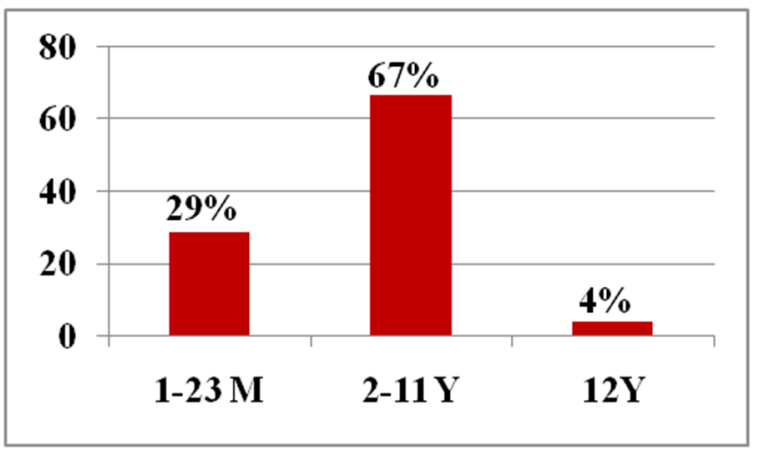

Fig. 1: Age wise classification

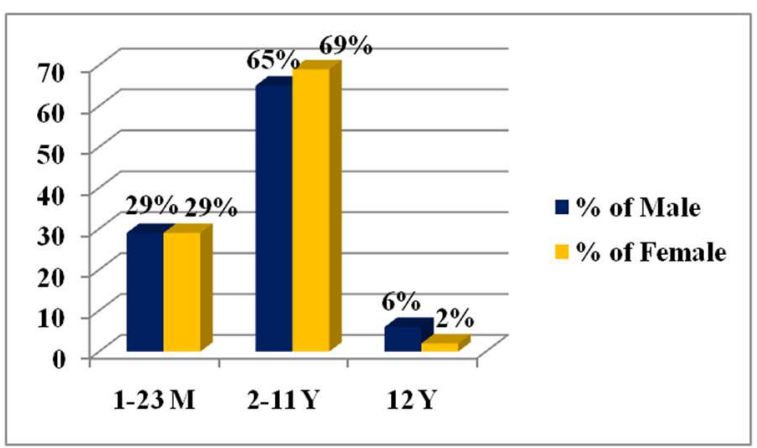

Fig. 2: Gender wise distribution

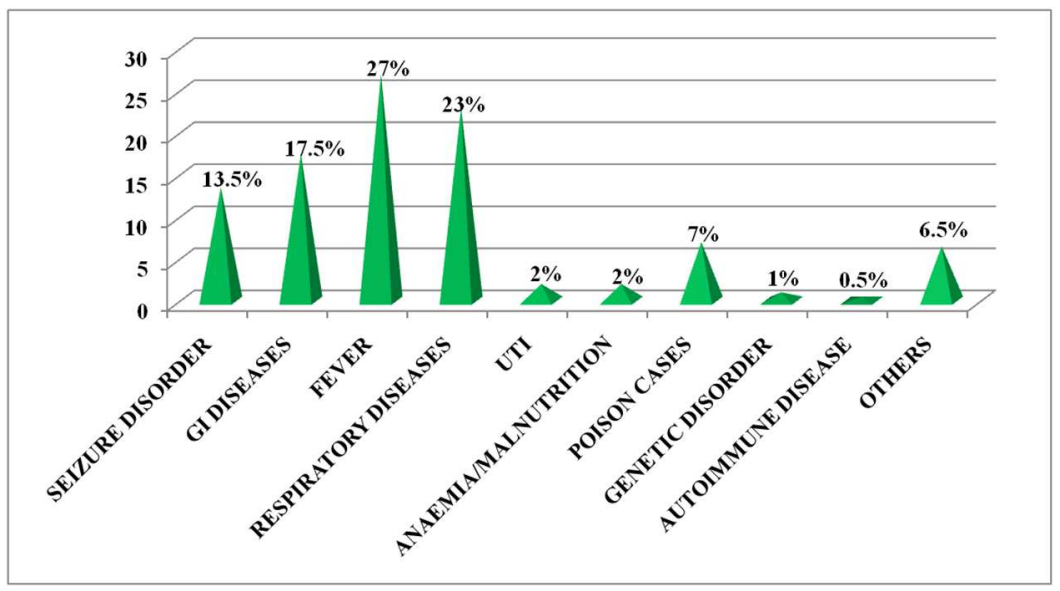

Fig. 3: Distribution of diseases 
Table 10: Assessment of prescribing indicators

\begin{tabular}{lll}
\hline Prescribing indicators assessed & Average/percentage & WHO standard \\
\hline Average number of drugs per encounter & 4.29 & $\leq 2$ \\
Percentage of drugs prescribed by generic name & $97.78 \%$ & $100 \%$ \\
Percentage of encounters with antibiotics & $90.5 \%$ & $<30 \%$ \\
Percentage of encounters with injections & $97.5 \%$ & $<20 \%$ \\
Percentage of drugs from essential drug list & $100 \%$ & $100 \%$ \\
\hline
\end{tabular}

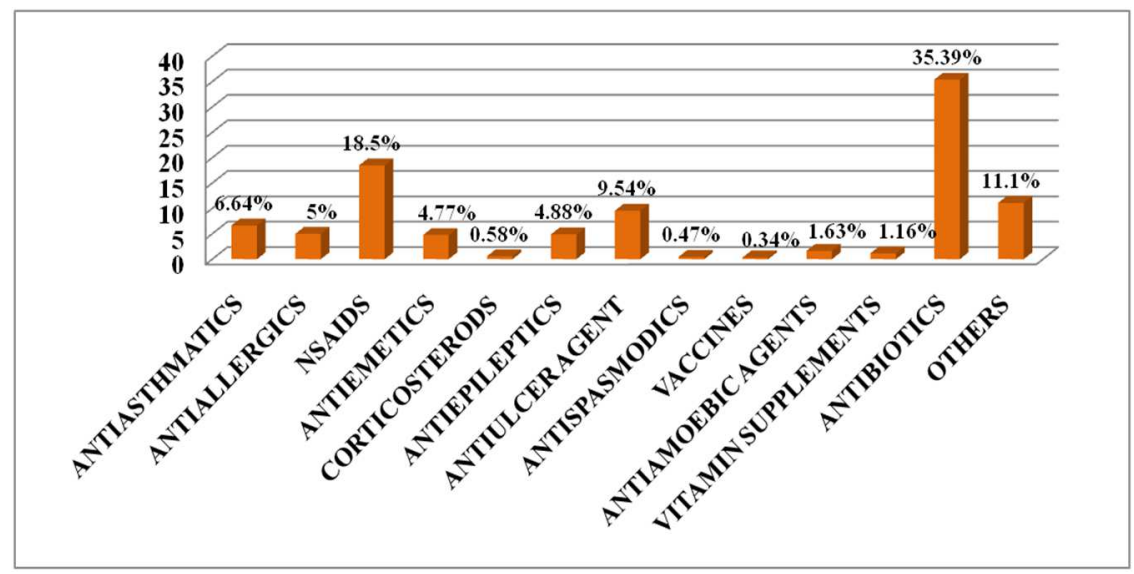

Fig. 4: Medicines prescribed to paediatrics

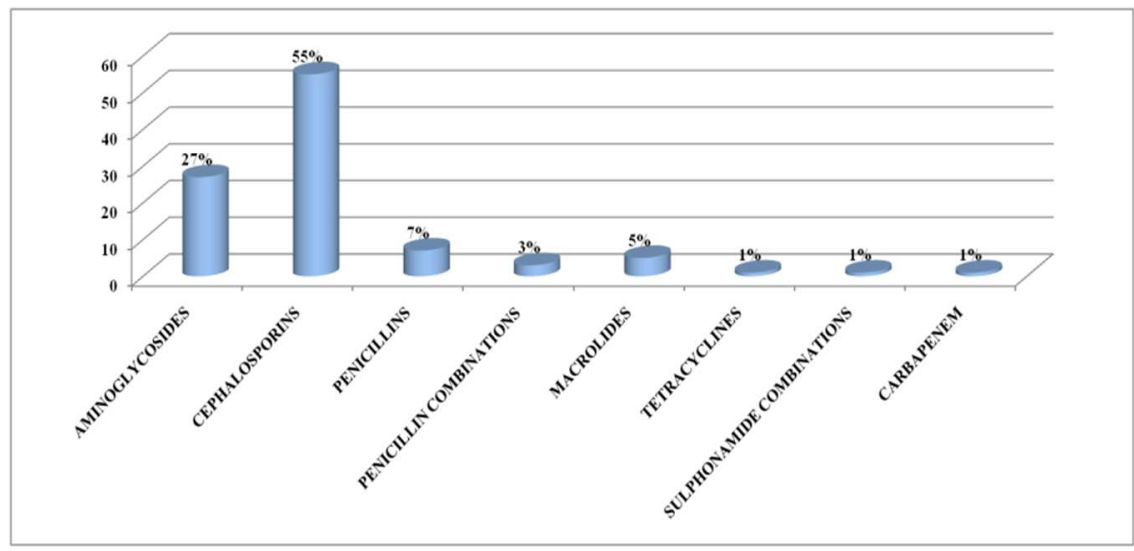

Fig. 5: Antibiotics prescribed

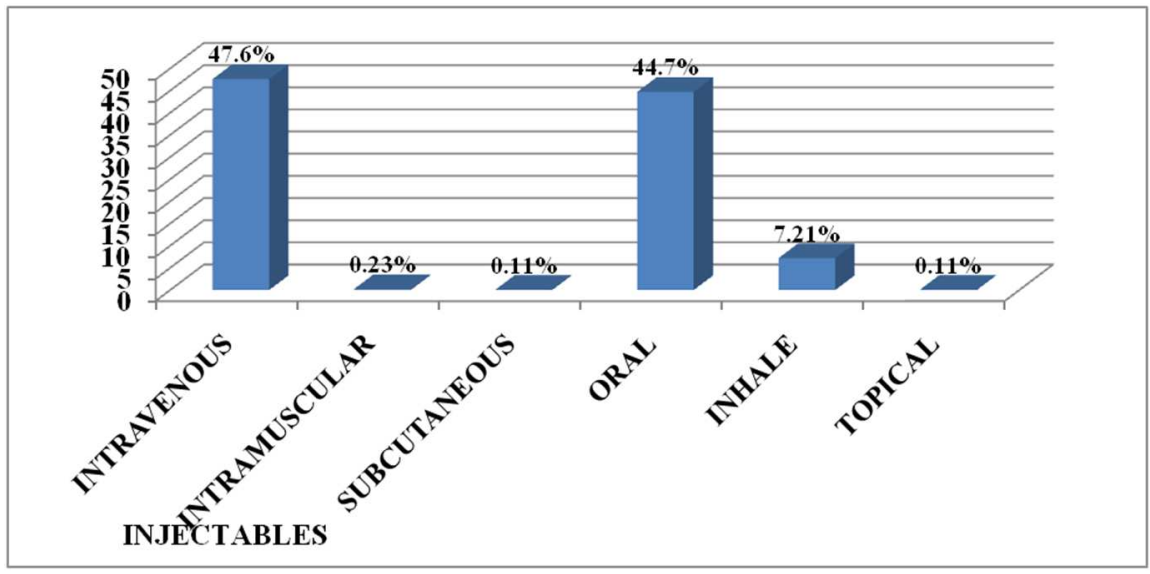

Fig. 6: Route of administration 


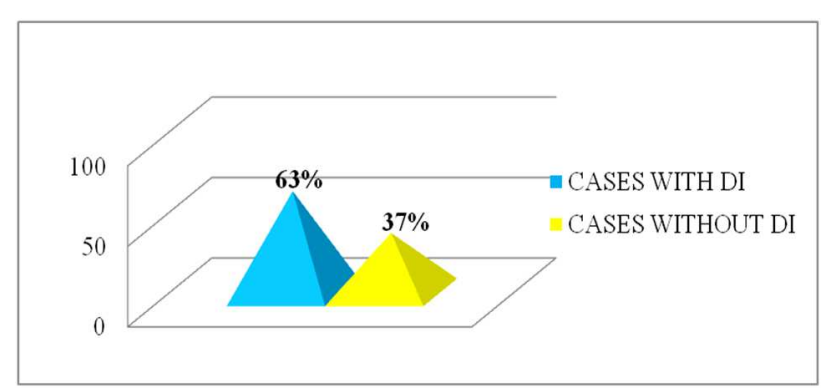

Fig. 7: Drug-drug interaction

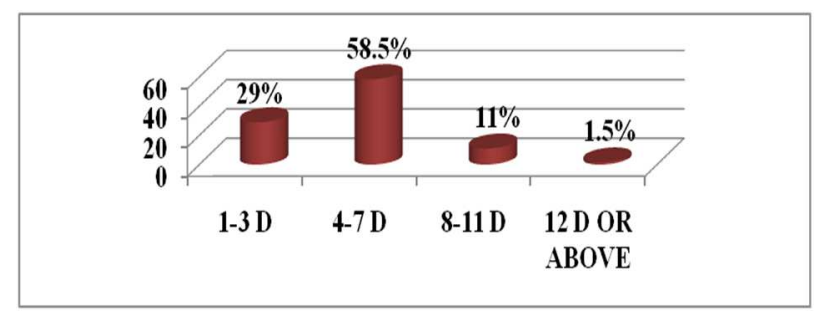

Fig. 8: Duration of hospital stay

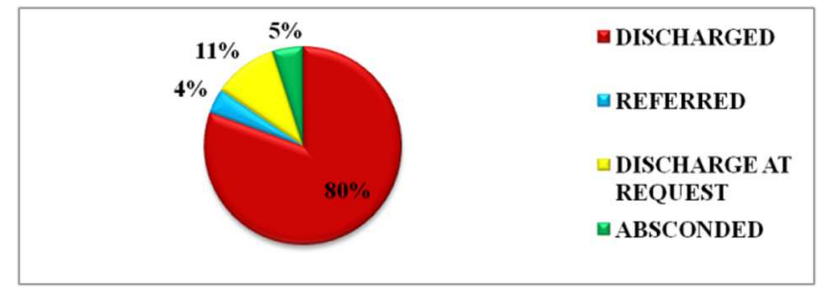

Fig. 9: Patient outcomes

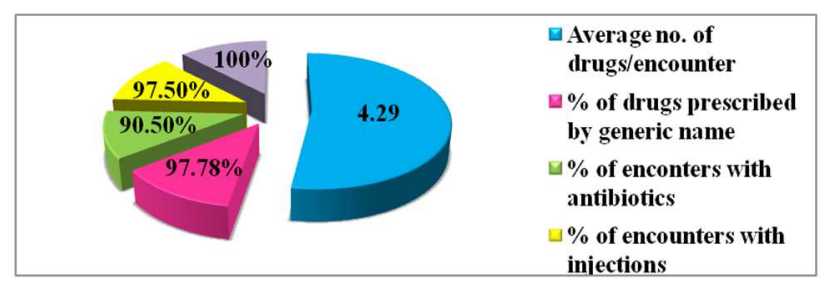

Fig. 10: WHO prescribing indicators

\section{DISCUSSION}

The paediatrics were considered to be infants and toddlers (1 mo-23 mo), children (2-11 y) and adolescents (12 y) according to age group and also by considering inclusion criteria of this study. Adolescent patients are $8(4 \%)$, found to be significantly less when compared to infants and toddlers 58 (29\%) and children 134 (67\%).

The number of male patients (65\%) and female patients (69\%) was found to be significantly high in the age group 2-11 y (children) when compared to the number of male patients $(6 \%)$ and female patients (2\%) in the age group of $12 \mathrm{y}$ (adolescents). An equal number of male and female patients $(29 \%)$ was observed in the age group $1 \mathrm{mo}-23 \mathrm{mo}$ (infants and toddlers).

The majority of paediatric patients were taken to the hospital due to fever and it was 54 (27\%). Second comes respiratory disease 46 (23\%). Gastrointestinal disorder was found to be 35 (17.5\%) and then comes seizure disorder 27 (13.5\%). Paediatric patients admitted with poisoning cases were $14(7 \%)$ and with genetic disorder is $1(0.5 \%)$. Paediatrics admitted due to urinary tract infection and anaemia/malnutrition were found to be equal $4(2 \%)$.
Only $1(0.5 \%)$ is admitted due to autoimmune disease and $13(6.5 \%)$ belong to other diseases. An earlier study reported that respiratory system disease $(25.6 \%)$, was most commonly seen, followed by fever (19.2\%), GIT (18.4\%) and hepatitis (8.8\%) disorders [6].

The highest prescribed drug class is antibiotics 304 (35.39\%). Second commonly prescribed drugs belong to nonsteroidal antiinflammatory drugs (NSAIDs) 159 (18.5\%). 57 (6.64\%) number of drugs prescribed are anti-asthmatics, $43(5 \%)$ are anti-allergic, 41 $(4.77 \%)$ are antiemetic, $5(0.58 \%)$ are corticosteroids, $42(4.88 \%)$ number of drugs belong to anti-epileptic, $82(9.54 \%)$ are anti-ulcer agents, $14(1.63 \%)$ are anti-amoebic agents, $10(1.16 \%)$ are vitamin supplements and $4(0.47 \%)$ are antispasmodic. The least prescribed drugs are vaccines $3(0.34 \%)$. The rest of drugs $95(11.1 \%)$ are categorized as others. A previous study reported that analgesic drugs were the most frequently prescribed therapeutic class of drugs (26.9\%) [12].

Among antibiotics, cephalosporin $167(55 \%)$ are the most prescribed and then comes aminoglycosides 83 (27\%). 22 (7\%) number of drugs is penicillin, $15(5 \%)$ are macrolides and $11(3 \%)$ are penicillin combinations. Equal number of drugs 2 (1\%) are prescribed as tetracyclines, carbapenem and sulphonamide combinations. In a study, it was found that the most frequently prescribed group of antibacterial were cephalosporin (62\%) followed by penicillin [13].

The majority of drugs were administered in injectable form 412 $(47.94 \%)$, in which the intravenous route is most commonly administered 409 (47.6\%). Only 2 (0.23\%) of drugs are administered via intramuscular route and $1(0.11 \%)$ is given via subcutaneous route. Oral route is contributing around $384(44.7 \%)$. Inhalation route contributes around $62(7.21 \%)$ and topical was only $1(0.11 \%)$. In another study it was found that $85.6 \%$ of medications are oral medications, $4.4 \%$ of cases consist of inhalational drugs, $8.6 \%$ of cases are topical drugs and $1.3 \%$ are suppositories or pessesaries [14].

$27(13.5 \%)$ number of prescriptions was found to be there with drug duplication and the remaining $173(86.5 \%)$ were identified as cases without drug duplication as per drugs. com website. $126(63 \%)$ prescriptions are cases with drug interaction and $73(37 \%)$ number of prescriptions are found to be without drug interactions.141 (72.31) number of moderate drug interactions and 54(27.69\%) number of minor drug interactions were found by analysing the prescription. Major drug interactions are not reported in case drugfood interaction $184(92 \%)$ prescriptions have no drug-food interactions and only $16(8 \%)$ number of prescriptions have reported drug-food interactions. Utilizing drugs. com website it was determined that $155(77.5 \%)$ numbers are cases without contraindications and $45(22.5 \%)$ are cases with contraindications.

While considering the comorbidity $69(34.5 \%)$ prescriptions out of 200 are having 1 comorbidity, $9(4.5 \%)$ are prescriptions with 2 co morbidity and only $2(1 \%)$ are prescriptions with $\geq 3$ comorbidity. Majority of the prescriptions $117(58.5 \%)$ show a duration of hospital stay for 4-7 d, 58 (29\%) number of patients stayed in the hospital for 1-3 d, $22(11 \%)$ number of patients were stayed for a period of $8-11 \mathrm{~d}$ and $3(1.5 \%)$ patients were stayed for 12 or more days. $161(80.5 \%)$ number of patients were discharged after completion of treatment and $8(4 \%)$ were referred for better treatment. $21(10.5 \%)$ were discharged at the request and $10(5 \%)$ were absconded during the study.

An average number of drugs per prescription were 4.29 which is greater than double the average number (i.e., 2) recommended by the world health organisation (WHO). The increased value indicates the poly-pharmacy practice in this hospital. However, this cannot be considered irrational poly-pharmacy practice as there is need of empirical therapy till definitive diagnosis becomes clear and secondly for management of acute-life threatening conditions. Most of the patients require two or more drugs and here $52(26 \%)$ patients have received 5 drugs during the hospital stay, which is the highest.

The percentage of drugs prescribed by generic (international nonproprietary) name is 97.78 in this study but according to WHO 
standards the optimal value is $100 \%$. By using the generic name in the prescriptions, the chance of duplication of drug products can be avoided. Example: the prescribing of albuterol and duolin (levalbuterol/ipratropium) at a time.

Percentage of encounters with an antibiotic prescribed was $90.5 \%$ which is thrice greater than WHO standard $<30 \%$. This indicates an increased use of antibiotics in paediatric population. However, the increased use of antibiotics in paediatrics is caused by severe infections, prophylactic treatment for infections and for doubtful secondary bacterial infections in viral diseases. The lack of proper or definitive diagnosis is also a reason for increased antibiotic use.

Prescribing of injections is not within the WHO recommended range $<20 \%$ and it was far higher showing $97.5 \%$. However, this increased prescribing of injection is not irrational as the recommended range is for outpatients. Due to the severity of illness, unavailability of oral and other dosage forms for a particular drug and to increase medication adherence injection formulation may be prescribed more. Nevertheless, the overuse of injections may lead to economic cost and health hazards. In a previous study it was found that average number of drugs per prescription as 4 and percentage of drugs prescribed by generic name as $45 \%$. The percentage of antibiotics prescribed was $33 \%$ and percentage of encounters with an injection in that study was $30.6 \%$ [6].

In this study, it was found that the prescribing practice in paediatric ward is in complete adherence to national essential drug list/formulary.

The mean value of Index of Rational Drug Prescribing (IRDP) was 3.09 which are very close to WHO optimal value of 5 . The optimal index for all indicators was fixed at 1 . Values closer to 1indicates the rational drug use.

\section{CONCLUSION}

This study demonstrates that prescription by generic name and prescribing drugs from EDL and free government supply of health care is encouraging findings. Poly-pharmacy and overuse of antibiotics are the areas to worry. Prescribing antibiotics to paediatric patients based on empirical therapy without culture sensitive test may lead to antibiotic resistance and health issues in the future.

It is rational to choose the intravenous route in the paediatric patient with age less than 14 , who are admitted and who are in need of emergency care. Hence, prescribing of injections is not within the WHO recommend range $<20 \%$.

Sticking to state standard treatment guidelines in the light of WHO/INRUD for paediatrics and awareness among paediatricians will promote more rational prescribing.

\section{LIMITATION OF THE STUDY}

The study was conducted in a hospital in a rural area and only 200 paediatrics have participated in the study. As a result, we could not have a large sample size which could increase our work significance.

\section{AUTHORS CONTRIBUTIONS}

R. Senthilselvi and V. Ganesan designed and conceived the study. Priya Visuvasam and R. Senthilselvi analyzed, interpreted the data and drafted and revised the article while Linta Sathyan and M. Boopana participated in data collection and article revision.

\section{CONFLICTS OF INTERESTS}

All authors have none to declare

\section{REFERENCES}

1. World Health Organization. Introduction to drug utilization research. Geneva: WHO; 2003.

2. World Health Organization. The rational use of drugs. Report of Conference of Experts: WHO; 1985.

3. Meena A, Kumar V, Agarwal A. Evaluation of drug utilization pattern in indoor patients of medicine department at tertiary care teaching hospital in Southern Rajasthan. Int J Pharm Sci Res 2016;7:3835-40.

4. Kumar MA, Ram KT, Ramasamy C. Cross-sectional prospective study on drug utilization in an outpatient paediatric department of tertiary care teaching hospital. Global J Pharmacol 2013;7:99-102.

5. Ramanath KV, Balaji BV. Study of outpatient prescription pattern of antibiotics in paediatric populations of two hospitals. Arch Pharm Pract 2013;4:21-7.

6. Sireesha G, Thomas JC, Patil PB, Doddayya H. A prospective study on medication prescribing pattern for paediatrics in a tertiary care teaching hospital. World J Pharm Pharm Sci 2016;5:654-66.

7. Ashraf H, Handa S, Khan NA. Prescribing pattern of drugs in outpatient department of a childcare centre in Moradabad city. Int J Pharm Sci Rev Res 2010;3:1-5.

8. The importance of pharmacovigilance; safety monitoring of medicinal products. Geneva, World Health Organization; 2002.

9. Abidi A, Gupta S, Kansal S. Prescription auditing and drug utilization pattern in tertiary care teaching hospital in western UP. Int J Basic Clin Pharmacol 2012;1:184-90.

10. Kearns GL, Abdel-Rahman SM, Alander SW, Blowey DL, Leeder JS, Kauffman RE. Developmental pharmacology-drug disposition, action, and therapy in infants and children. N Engl J Med 2003;349:1157-67.

11. World Health Organization. Promoting Safety of Medicines for Children. Geneva WHO; 2007.

12. Santos LD, Heineck I. Drug utilization study in paediatric prescription of a university hospital in southern brazil: offlabel, unlicensed and high-alert medications. Farm Hosp 2012;36:180-6.

13. Gupta A, Maheshwari P, Dokania KK, Tambe JJ. Drug utilization pattern in sick paediatric patients: analysis for rationality and other aspects. Int J Basic Clin Pharmacol 2017;6:2815-21.

14. Murthy NV, Faisal EM, Kumar RS. Assessment of drug prescription pattern in paediatric patients. J Pharm Sci Res 2017;9:81-4. 